\title{
Clinical effectiveness of ranibizumab and conbercept for neovascular age-related macular degeneration: a meta-analysis
}

This article was published in the following Dove Press journal:

Drug Design, Development and Therapy

Luping Wang'

Canwei Zhang ${ }^{2}$

Rui Hua ${ }^{3}$

'Department of Ophthalmology, University of Bonn, Bonn, Germany;

${ }^{2}$ Department of Ophthalmology, Weifang Eye Hospital, Weifang,

People's Republic of China;

${ }^{3}$ Department of Ophthalmology, First Hospital of China Medical University, Shenyang, People's Republic of China

Correspondence: Rui Hua Department of Ophthalmology, First Hospital of China Medical University, No I55, Nanjingbei Street, Heping District, Shenyang, Liaoning Province, People's Republic of China Tel +86 I384058 3355

Fax +862483282630

Email woodshua@I26.com
Introduction: To assess the ocular efficacy of intravitreal ranibizumab and conbercept injection in patients with neovascular age-related macular degeneration.

Materials and methods: We searched PubMed, Wed of Science, Cochrane Library, EMBASE, Google Scholar, Medline, China National Knowledge Infrastructure, and WANFANG DATA databases, up to June 20, 2018. We also searched abstracts and clinical study presentations at meetings as well as trial registries; we contacted authors of included studies if questions arose. Eligibility criteria for selection of studies were randomized controlled trials and retrospective trials that compared ranibizumab with conbercept for treatment of neovascular age-related macular degeneration.

Results: Eight randomized controlled trials and four retrospective studies were included with a total of 853 patients. Best-corrected visual acuity after loading dosage was improved in the conbercept group, compared with the ranibizumab group (weighted mean difference: $-0.04 ; 95 \% \mathrm{CI}:-0.07$ to $0.00 ; P=0.04)$. There was a significant difference between conbercept and ranibizumab therapy with respect to unchanged or recurrent leakage of choroidal neovascularization (OR: $0.46 ; 95 \%$ CI: $0.24-0.88 ; P=0.02$ ). No significant differences were observed in central macular thickness (weighted mean difference: $-2.92 ; 95 \% \mathrm{CI}:-9.00$ to $3.17 ; P=0.35$ ), complete and partial closure of leakage of choroidal neovascularization (complete closure, $P=0.70$; partial closure, $P=0.35$ ), or number of injections (weighted mean difference: $0.42 ; 95 \% \mathrm{CI}:-0.46$ to $1.29 ; P=0.35$ ) between the conbercept and ranibizumab groups at the end of the follow-up periods.

Conclusion: Pooled evidence confirmed that conbercept was superior to ranibizumab with respect to visual gain after treatment. Additional studies with long-term follow-up are needed to support our conclusion.

Keywords: age-related macular degeneration, best-corrected visual acuity, central macular thickness, choroidal neovascularization, vision loss

\section{Introduction}

Age-related macular degeneration (AMD), which comprises $8.7 \%$ of all cases of blindness, is the third leading cause of irreversible vision loss worldwide and the most common cause of blindness in people over 50 years of age. ${ }^{1}$ Advanced AMD includes neovascular (wet or exudative) AMD (nAMD), which is characterized by choroidal neovascularization (CNV) and proliferation of fibrous tissue. ${ }^{2}$ Notably, wet AMD constitutes $10 \%-15 \%$ of all cases of AMD but is responsible for $>90 \%$ of severe visual loss from AMD. ${ }^{3}$ Patients with nAMD can experience sudden and severe central vision loss within days or weeks, primarily caused by CNV. ${ }^{4-6}$ Vascular endothelial growth factor (VEGF) has been identified as a principle mediator of CNV in the pathogenesis 
of AMD. Thus far, the application of anti-VEGF drugs has been the mainstay of nAMD treatment. Anti-VEGF agents, including ranibizumab (Lucentis ${ }^{\circledR}$, Genentech Inc., South San Francisco, CA, USA), bevacizumab, and aflibercept, substantially reduce visual loss in the treatment of nAMD. ${ }^{7}$

Ranibizumab is a recombinant, humanized, monoclonal immunoglobulin G1 (IgG1) $\kappa$ isotype antibody antigenbinding fragment (Fab), which can neutralize all isoforms of VEGF-A. It was approved for the treatment of nAMD by the US Food and Drug Administration (FDA) in 2006. ${ }^{8-10}$ The newest anti-VEGF drug, conbercept (or KH902, Lumitin, Chengdu Kang Hong Biotech Co, Ltd., Sichuan, People's Republic of China), is a recombinant fusion protein composed of extracellular domain 2 of VEGFR-1 and extracellular domains 3 and 4 of VEGFR-2 (KDR-d4), combined with the Fc portion of human IgG1. Conbercept is similar in structure to aflibercept, an anti-VEGF agent that binds to all isoforms of VEGF-A, VEGF-B, and placental growth factor (PlGF); additionally, conbercept exhibits a higher affinity to VEGF because of the addition of the fourth Ig-like domain of VEGFR-2 in the Fab fragment. ${ }^{11}$

In clinical practice, conbercept is effective in some patients who are nonresponsive to ranibizumab and bevacizumab. ${ }^{12,13}$ In preclinical trials, the binding affinity of conbercept for VEGF was reported to be significantly greater than that of ranibizumab. ${ }^{14}$ Conbercept was approved for the treatment of nAMD by the China State FDA in December 2013. However, the drug has not yet reached other markets. This meta-analysis aimed to compare the ocular efficacy of conbercept and ranibizumab in the treatment of nAMD from the perspective of studies from People's Republic of China.

\section{Materials and methods Literature search}

A systematic search was performed to identify relevant studies comparing ranibizumab with conbercept for the treatment of nAMD by using the following databases: PubMed, Wed of Science, Cochrane Library, EMBASE, Google Scholar, Medline, China National Knowledge Infrastructure, and WANFANG DATA. The search included all published trials up to June 20, 2018, with the following Medical Subject Heading terms: "Macular Degeneration or Age-Related Macular Degeneration or AMD or ARMD or nAMD," and "ranibizumab or Lucentis," and "conbercept or KH902 or Lumitin." No language restrictions were used in the search. Furthermore, the "related articles" function was used to broaden the search, and all relevant studies were included for further screening.

\section{Inclusion criteria and exclusion criteria}

Trials were included if they met the following criteria: 1) randomized controlled trials (RCTs) and/or non-RCTs, 2) studies that included the comparison of ranibizumab with conbercept, 3) patients with AMD that required anti-VEGF therapy, and 4) studies that included at least one outcome of interest mentioned below with relative data reported or able to be calculated: number of injections of ranibizumab and conbercept, best-corrected visual acuity (BCVA), and central macular thickness (CMT) on optical coherence tomography. Trials were excluded if any of the following conditions were met: 1) patients had other diseases, such as diabetic retinopathy, 2) no outcomes of interest were reported, or calculating or extrapolating the necessary data for either ranibizumab or conbercept from the published results was impossible, and/or 3) reports were duplicated.

\section{Data extraction and outcomes of interest}

Two independent reviewers (RH and LPW) searched the literature, assessed the quality of trials, and extracted the following data with a standardized form: first author name, year of publication, country, study interval, study design, number of patients who underwent ranibizumab or conbercept therapy, follow-up duration, and outcomes of interest. All BCVA values were converted into log minimum angle of resolution (ie, LogMAR) for analysis. All discrepancies regarding eligibility and data extraction were resolved by discussion among all authors until a consensus was reached.

\section{Study quality and level of evidence}

The levels of evidence of included studies were rated by two reviewers, according to criteria used by the Centre for Evidence-Based Medicine in Oxford, UK. ${ }^{15}$ The reviewers independently assessed the quality of the studies, and any disagreement was resolved by consensus.

\section{Statistical analyses}

The present meta-analysis was conducted according to the recommendations of the Cochrane Collaboration, as well as the Quality of Reporting of Meta-analyses guidelines. ${ }^{16}$ All the statistical analyses were performed by using RevMan 5.3 (Cochrane Library Software, Oxford, UK). Weighted mean differences (WMDs) were used for continuous variables; odds ratios (ORs) were used for dichotomous variables. The CIs were set at $95 \%$.

All pooled estimates were determined by using the $z$ test; $P$-values $<0.05$ were considered statistically significant. The degree of heterogeneity among included studies was assessed by using the chi-squared-based $Q$ test and the $I^{2}$ test; 
heterogeneity was defined by $I^{2}>50 \%$ and $P<0.05$. When the evidence indicated that interstudy heterogeneity existed, a random-effects model was used; otherwise, a fixed-effects model was adopted. Sensitivity analysis was performed by omission of specific studies. Variables were pooled when outcomes were reported by three or more studies in the overall meta-analysis. Publication bias was assessed by using a funnel plot.

\section{Results}

\section{Literature review process}

After screening, a total of 12 studies ${ }^{17-28}$ with a total of 853 participants were included in the present meta-analysis. The studies included eight RCTs $\mathrm{s}^{20-24,26-28}$ and four retrospective studies, ${ }^{17-19,25} 433$ and 420 patients received injections of ranibizumab and conbercept, respectively. Figure 1 shows a flow diagram of the study procedure; Table 1 shows the characteristics of the included studies.

\section{BCVA}

No significant difference was observed in BCVA before treatment between the conbercept and ranibizumab groups (WMD: $0.01 ; 95 \% \mathrm{CI}:-0.02$ to $0.03 ; P=0.65$ ). However, after 3 months treatment with conbercept or ranibizumab, BCVA significantly differed between the two groups (WMD: $-0.04 ; 95 \% \mathrm{CI}:-0.07$ to $0.00 ; P=0.04$ ). Studies by Cai and Peng ${ }^{17}$ and Cui et al ${ }^{18}$ were not included because they only showed improvement of vision, rather than BCVA, after treatment with conbercept or ranibizumab. Patients treated with monthly injections of conbercept experienced greater improvement of BCVA from baseline compared with patients treated with ranibizumab. Figure 2 shows the source data describing changes of BCVA in the included studies.

\section{CMT}

Average CMTs were detected on optical coherence tomography images at the start and end of the follow-up period in the conbercept and ranibizumab groups. No significant differences were observed in the average CMT before treatment (WMD: $-2.62 ; 95 \% \mathrm{CI}:-9.92$ to $4.68 ; P=0.48$ ) and after treatment (WMD: $-2.92 ; 95 \% \mathrm{CI}:-9.00$ to 3.17 ; $P=0.35$ ) between the conbercept and ranibizumab groups (Figure 3).

\section{Leakage of CNV}

No significant differences were observed in the rate and degree of CNV recovery between the conbercept and ranibizumab groups, in complete closure (OR: 1.10; 95\% CI: $0.68-1.79 ; P=0.70)$ or partial closure (OR: $1.26 ; 95 \% \mathrm{CI}$ : $0.78-2.03 ; P=0.35$ ) (Figure 4 ). However, there was a significant difference between the two groups in unchanged or recurrent leakage of CNV (OR: 0.46; 95\% CI: 0.24-0.88; $P=0.02$ ) (Figure 4).

\section{Number of injections}

No statistical difference was observed in the mean number of injections between the conbercept and ranibizumab groups (WMD: $0.42 ; 95 \%$ CI: -0.46 to $1.29 ; P=0.35$ ) (Figure 5).

\section{Sensitivity analysis and publication bias}

Heterogeneity was apparent in injection numbers $(P<0.00001$, $I^{2}=94 \%$ ). Therefore, sensitivity analysis was performed, in

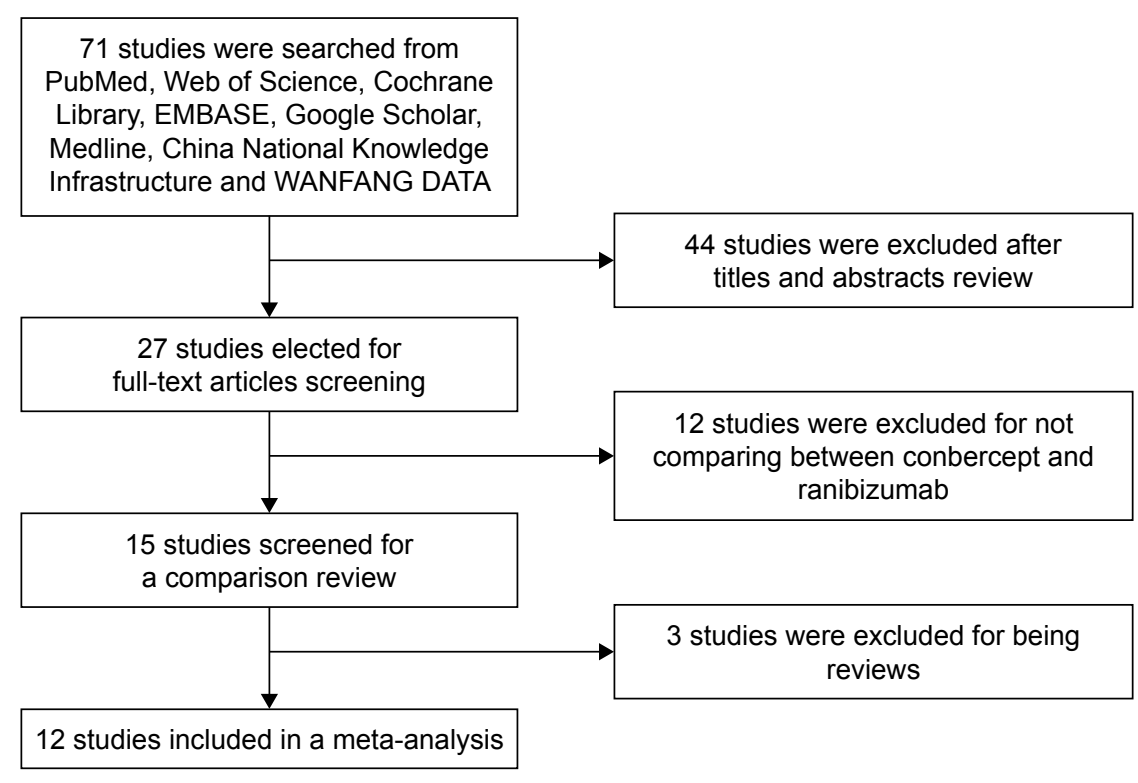

Figure I Flowchart showing the selection of studies for meta-analysis. 
Table I Characteristics of included studies

\begin{tabular}{|c|c|c|c|c|c|c|}
\hline Study & Country & $\begin{array}{l}\text { Study } \\
\text { interval }\end{array}$ & Study design & LOE $^{a}$ & Treatment regimen & $\begin{array}{l}\text { Number of patients, } \\
\text { conbercept/ } \\
\text { ranibizumab }\end{array}$ \\
\hline Cai and Peng, ${ }^{17} 2016$ & People's Republic of China & $2013-2015$ & Retrospective & $2 b$ & Monthly & $30 / 30$ \\
\hline Cui et al, ${ }^{18} 2018$ & People's Republic of China & $2014-2015$ & Retrospective & $2 b$ & As-needed & $83 / 85$ \\
\hline Huang et al, ${ }^{19} 2018$ & People's Republic of China & $2013-2016$ & Retrospective & $2 b$ & $\begin{array}{l}\text { Monthly for } 3 \text { months } \\
\text { then as-needed }\end{array}$ & $35 / 44$ \\
\hline Li et al, ${ }^{20} 2018$ & People's Republic of China & $2016-2017$ & $\mathrm{RCT}$ & $2 b$ & $\begin{array}{l}\text { Monthly for } 3 \text { months } \\
\text { then as-needed }\end{array}$ & $20 / 20$ \\
\hline Lv et al, ${ }^{21} 2016$ & People's Republic of China & $2013-2015$ & $\mathrm{RCT}$ & $2 b$ & $\begin{array}{l}\text { Monthly for } 3 \text { months } \\
\text { then as-needed }\end{array}$ & $42 / 42$ \\
\hline Niu et al, 22016 & People's Republic of China & $2014-2015$ & RCT & $2 b$ & Monthly & $20 / 20$ \\
\hline Yang, ${ }^{23} 2018$ & People's Republic of China & $2014-2016$ & RCT & $2 b$ & As-needed & $24 / 24$ \\
\hline Zhang and Zhao, ${ }^{24} 2016$ & People's Republic of China & $2014-2016$ & RCT & $3 b$ & Monthly & $25 / 25$ \\
\hline Zhang, ${ }^{25} 2017$ & People's Republic of China & $2015-2016$ & RCT & $2 b$ & Monthly & $20 / 20$ \\
\hline Zhang and Bai, ${ }^{26} 2017$ & People's Republic of China & $2014-2016$ & RCT & $3 b$ & Monthly & $49 / 49$ \\
\hline Zhao and $\mathrm{Bai}^{27}{ }^{2015}$ & People's Republic of China & $2013-2014$ & Retrospective & $2 b$ & $\begin{array}{l}\text { Monthly for } 3 \text { months } \\
\text { then as-needed }\end{array}$ & $30 / 31$ \\
\hline Zheng, ${ }^{28} 2017$ & People's Republic of China & $2013-2014$ & RCT & $2 b$ & $\begin{array}{l}\text { Monthly for } 3 \text { months } \\
\text { then as-needed }\end{array}$ & $42 / 43$ \\
\hline
\end{tabular}

Note: ${ }^{a}$ Based on US Preventive Services Task Force grading system.

Abbreviations: LOE, level of evidence; RCT, randomized control trial.

which specific studies were omitted and the remaining studies were analyzed to determine whether the results could have been markedly affected by a single study. Sensitivity analyses suggested that no individual study significantly affected the overall estimate of the numbers of injections. We concluded that the reasons for heterogeneity, other than clinical differences, such as different treatment regimen, could include small sample size, which was inadequate to accurately estimate heterogeneity. No significant heterogeneity was observed in the remaining seven measures (BCVA before treatment: $\chi^{2}=1.85, d f=4, P=0.76, I^{2}=0 \%$; BCVA after treatment: $\chi^{2}=3.87, d f=4, P=0.42, I^{2}=0 \%$; CMT before treatment: $\chi^{2}=2.64, d f=10, P=0.99, I^{2}=0 \%$; CMT after treatment: $\chi^{2}=9.13, d f=9, P=0.43, I^{2}=1 \%$; complete closure of leakage of CNV: $\chi^{2}=1.40, d f=3, P=0.71, I^{2}=0 \%$ : partial closure of leakage of CNV: $\chi^{2}=1.18, d f=3, P=0.76, I^{2}=0 \%$ ). A funnel

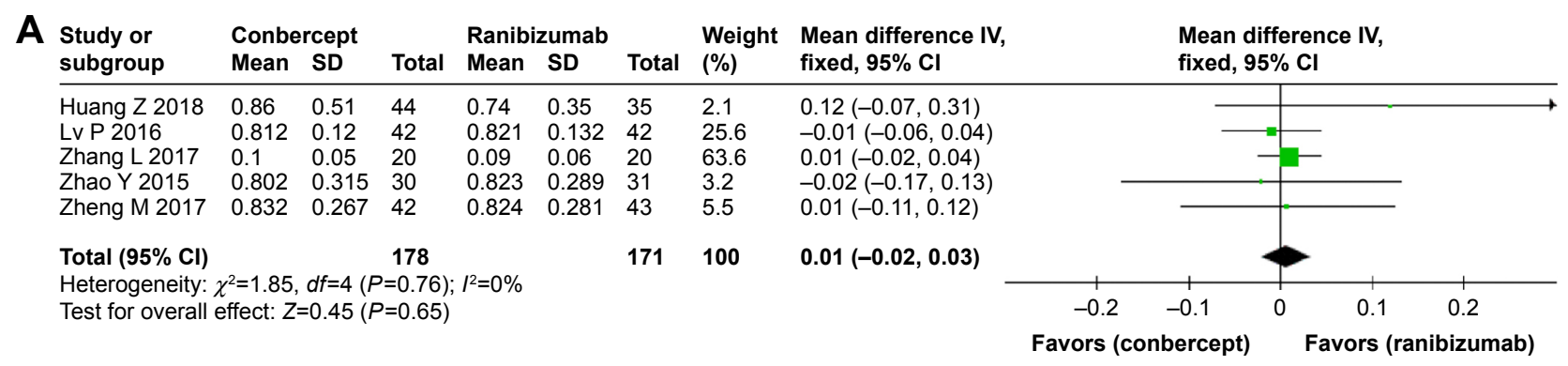

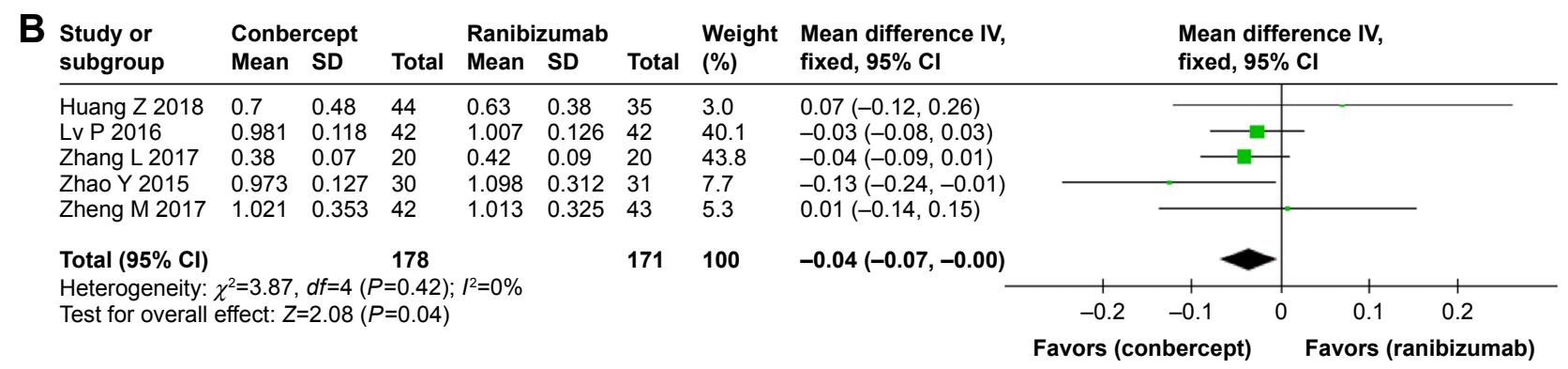

Figure 2 Forest plot and meta-analysis of BCVA.

Notes: (A) BCVA before treatment; (B) BCVA after treatment.

Abbreviation: BCVA, best-corrected visual acuity. 
A

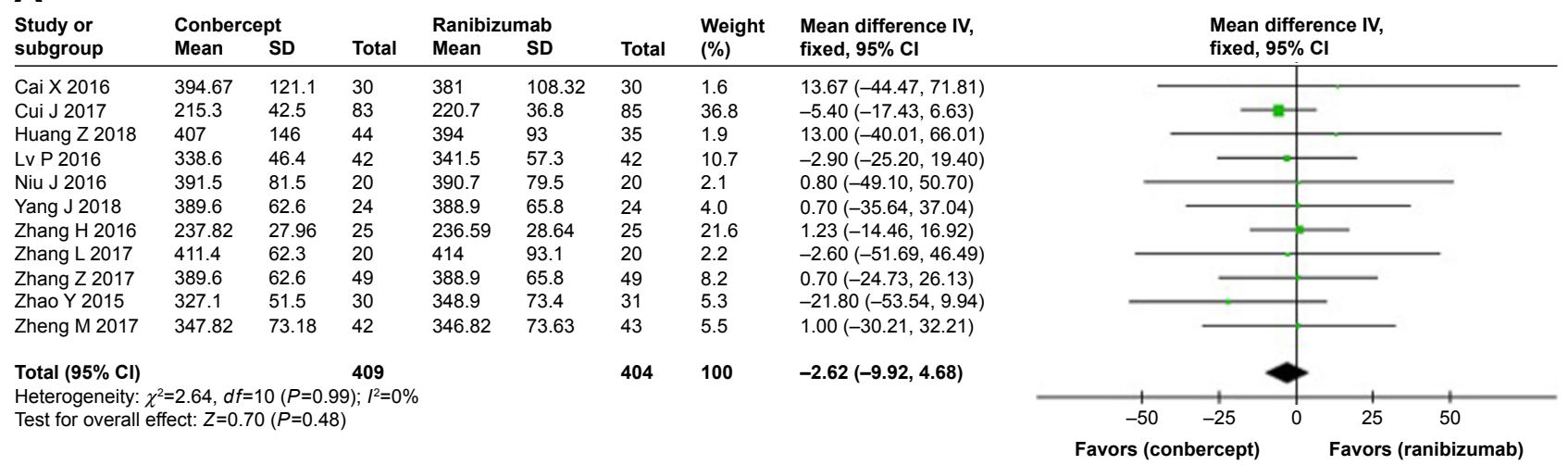

B

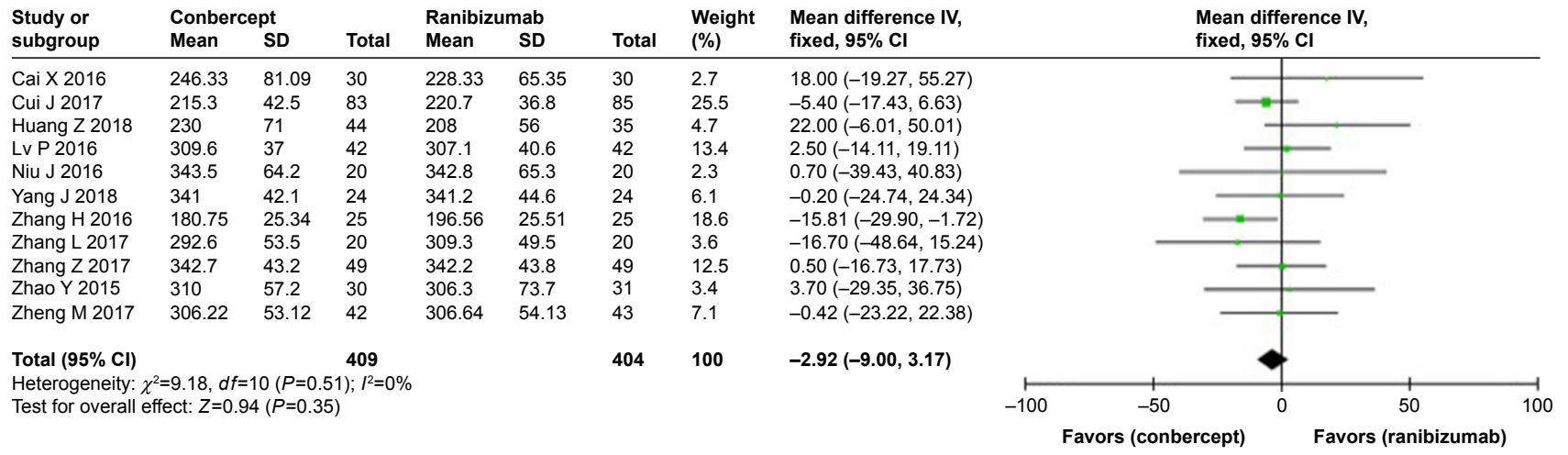

Figure 3 Forest plot and meta-analysis of CMT.

Notes: (A) CMT before treatment; (B) CMT after treatment.

Abbreviation: CMT, central macular thickness.

plot was used to assess the publication bias of the included studies, and no significant publication bias was found in any of the comparisons (Figure 6).

\section{Discussion}

In People's Republic of China, conbercept has been widely used as the first-line drug for the treatment of nAMD for nearly 5 years. Therefore, the systemic effects of conbercept during treatment of nAMD require great attention. The efficacies of ranibizumab and conbercept for nAMD have been reported separately by different groups; both drugs have been shown to significantly improve visual acuity in patients with nAMD. ${ }^{12,29}$ However, these data have not been systematically sorted, collected, and assessed. The present study provided further information regarding the ocular efficacy of conbercept and ranibizumab from a clinical perspective.

In this meta-analysis, no significant differences were noted in BCVA and CMT before treatment, which indicated that conbercept and ranibizumab cohorts showed no significant differences in baseline parameters. Interestingly, BCVA was significantly better in the conbercept group than in the ranibizumab group at the end of the follow-up period. Conbercept is similar to the anti-VEGF agent aflibercept (Eylea $^{\circledR}$, Regeneron Pharmaceuticals, Eastview, NY, USA), which binds to all isoforms of VEGF-A, VEGF-B, and PIGF. Indeed, this result is consistent with a study by Inoue et al, ${ }^{30}$ which reported superior BCVA at 6 months, compared with baseline, after aflibercept treatment. Additionally, Huang et $\mathrm{al}^{19}$ reported no significant difference in the visual improvement of patients with polypoidal choroidal vasculopathy between the conbercept and ranibizumab groups at 6 months; however, conbercept was superior to ranibizumab monotherapy in the regression of polyps. Moreover, Cui et al ${ }^{18}$ confirmed that conbercept and ranibizumab showed equivalent effects with respect to visual gain and reduction of central retinal thickness at 1 year, when administered according to a treat-and-extend protocol.

The above results confirmed superior BCVA after treatment in the conbercept group, compared with that in the ranibizumab group. However, no significant differences were observed in CMT between the two groups. The results of BCVA analysis seemed to be inconsistent with those 


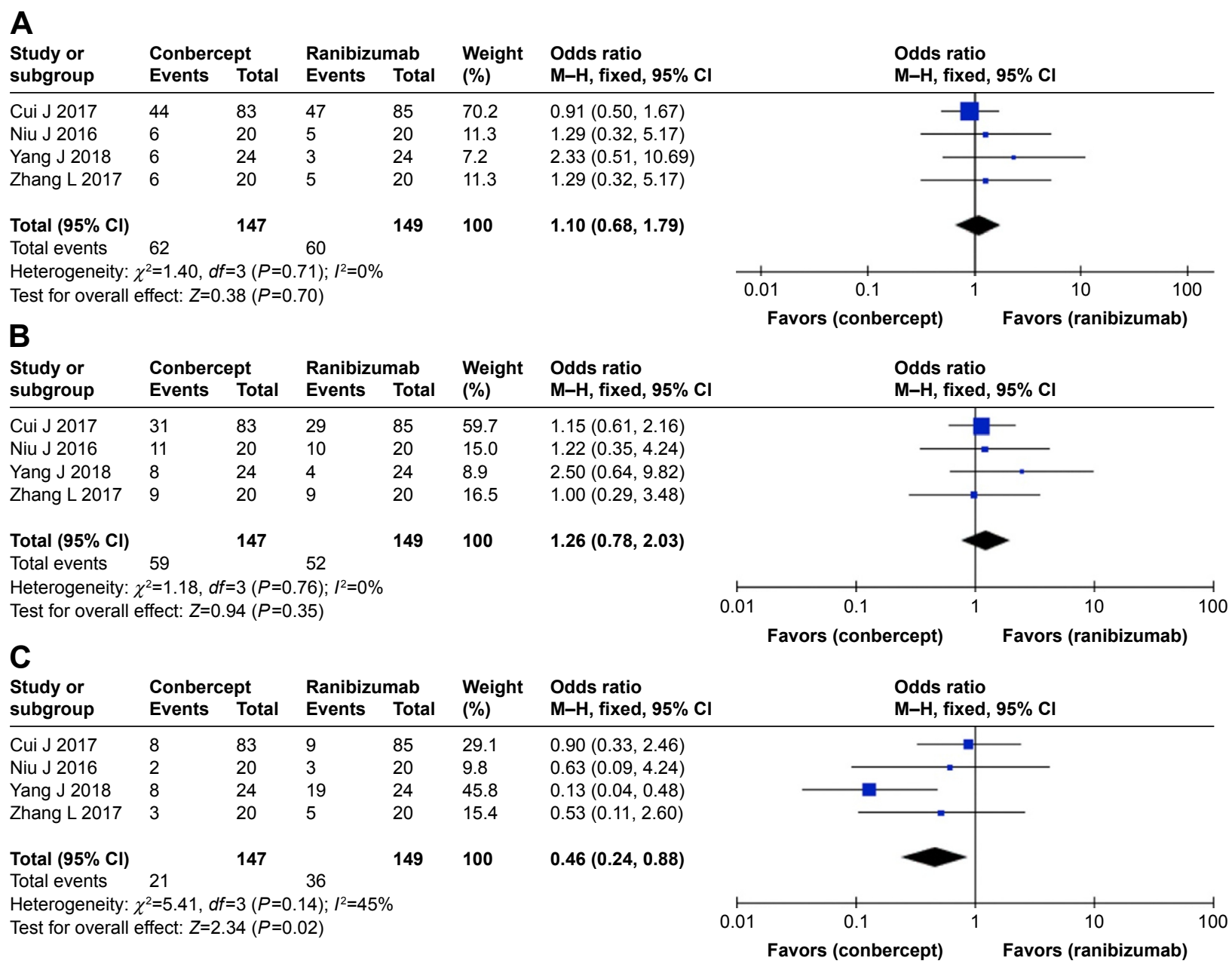

Figure 4 Forest plot and meta-analysis of leakage of CNV.

Notes: (A) Complete closure; (B) partial closure; (C) no change and recurrent exudative activity.

Abbreviation: CNV, choroidal neovascularization.

of CMT analysis. This inconsistency could be because CMT and BCVA are affected by the morphology, size, and level of macular edema, as well as the disruption of photoreceptors. ${ }^{31}$ The precursors of hard exudates in cysts located in the outer nuclear layer ${ }^{32}$ can invade the external limiting membrane and photoreceptors, resulting in photoreceptor degeneration and apoptosis; ${ }^{33}$ hydraulic pressure in the cysts also exerts an impact on the external limiting membrane and photoreceptors. ${ }^{33}$ Malfunctional Müller cell-derived VEGF causes pathological permeability of the barrier in the sensory retina under hypoxic or ischemic conditions. ${ }^{34}$ Pelosini et $\mathrm{al}^{35}$ concluded that the integrity of the cross-sectional area of retinal tissue between the plexiform layers in cystoid macular edema has a linear relationship

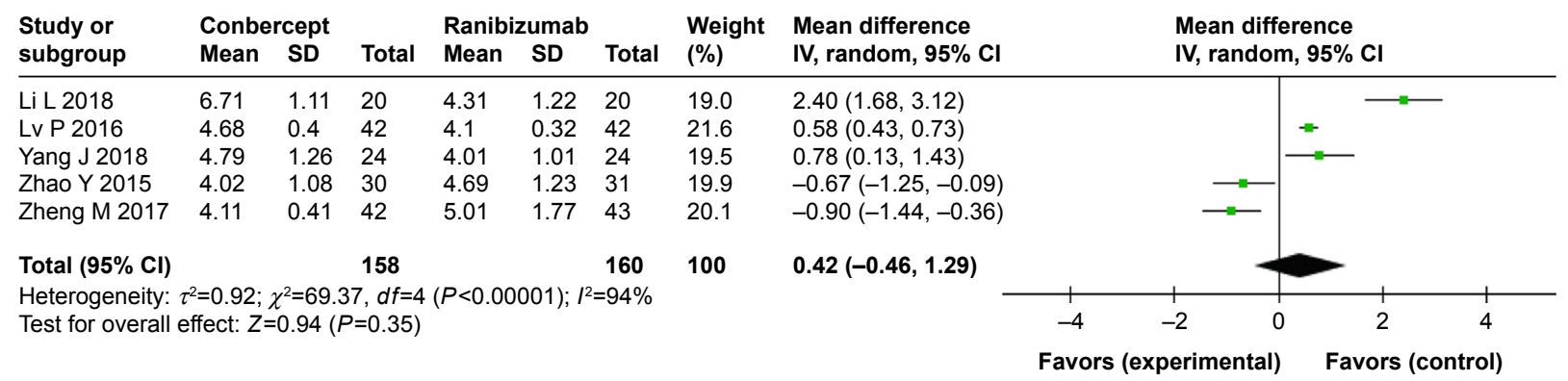

Figure 5 Forest plot and meta-analysis of the number of injections. Notes: Experimental group: conbercept; control group: ranibizumab. 


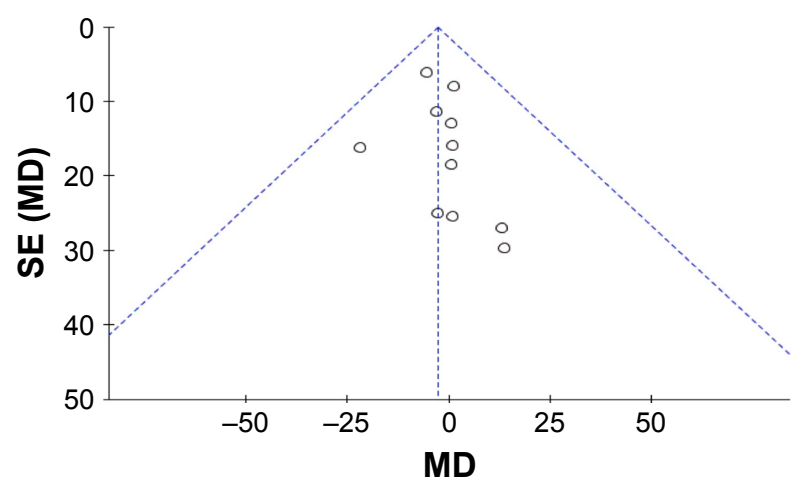

Figure 6 Funnel plot of publication bias.

with BCVA. Therefore, we inferred that intravitreal conbercept injection might improve visual function, which is related to the renewal of cells; reconstruct the retinal tissue; relieve damage to photoreceptors; and improve anatomical outcomes. Finally, anti-VEGF-A monotherapy induces the apoptosis of amacrine cells and bipolar cells (in the inner nuclear layer), as well as retinal ganglion cells, ${ }^{36-38}$ which may partially explain the difference in BCVA but similarity in CMT between the two groups.

We also found a significant difference in the unchanged or recurrent leakage of CNV between the two groups, although there were no differences in complete and partial closure. We speculated this might be because conbercept can suppress CNV by exerting both anti-VEGF and anti-inflammatory effects; conbercept can bind to all isoforms of VEGF-A, VEGF-B, and PIGF. ${ }^{39}$ Similarly, Qu et al ${ }^{40}$ reported that conbercept therapy led to complete regression of polyps in more than half of polypoidal choroidal vasculopathy patients, which is consistent with our results. No statistical difference was observed in the mean number of injections between the conbercept and ranibizumab groups.

The blocking potency of conbercept is similar to that of aflibercept $(8 \mathrm{pM})$ and is 38 - to 48 -fold greater than that of both ranibizumab and bevacizumab. ${ }^{41}$ Conbercept is a soluble receptor decoy that blocks all isoforms of VEGF-A, VEGF-B, VEGF-C, and PIGF, which has a high binding affinity for VEGF and a long half-life in the vitreous; its half-life is 4.2 days in rabbits. ${ }^{42}$ However, the half-life of ranibizumab is 2.88-2.89 days for $0.5 \mathrm{mg}$ in rabbits; it is 2.63 and 3.9 days for 0.5 and $2 \mathrm{mg}$, respectively in monkeys. ${ }^{41}$ If the half-life of anti-VEGF drugs were extended, the therapeutic effect could be extended, reducing the frequency of injections. The extended half-life indicates extended therapeutic effect and reduced injection times. ${ }^{43}$ Conbercept is a natural conjugator of multiple targets of VEGFR, and so it is reasonable to speculate that it might exhibit a longer duration of action. ${ }^{18}$ The concentrations of conbercept in the rabbit retina and choroid remained higher than the in vitro $50 \%$ inhibitory concentration value $(7 \mathrm{ng} / \mathrm{g})^{44}$ over 34 days, indicating that a single $0.5 \mathrm{mg}$ intravitreal injection may have an inhibitory effect against VEGF over the course of 81 days. $^{41}$

Moreover, domain 4 of VEGFR-2 exhibits a lower isoelectric point (PI). A prior study showed that a high positive charge of a fusion protein may lead to poor pharmacokinetic properties..$^{45}$ The addition of domain 4 to conbercept reduces its positive charge and may lead to reduced adhesion to the extracellular matrix. The PI of conbercept is $6.13-6.81,{ }^{14}$ which is lower than the PI of aflibercept (PI 8.82). ${ }^{45}$ The introduction of domain 4 may enhance its association with VEGF and prolong the half-life of the drug.

The current study has several limitations. First, conbercept has only recently been applied in clinical practice. Therefore, the data available from People's Republic of China are limited; this was our reason for inclusion of both RCTs and retrospective studies. Further studies with longterm follow-up periods and reports of curative effects are required to confirm whether the improvement in visual acuity at different time points as well as improvements in various anatomical outcomes are maintained over time. Second, further clinical research is required to compare the efficacy of conbercept with structurally similar anti-VEGF drugs, such as aflibercept $\left(\right.$ Eylea $\left.^{\circledR}\right)$, which has recently become commercially available in People's Republic of China. Moreover, personalized treatment also must be considered and thoroughly explored.

\section{Conclusion}

The results of this meta-analysis indicate that both conbercept and ranibizumab are effective choices for the treatment of AMD, although conbercept is superior with respect to visual gain.

\section{Acknowledgment}

This study was supported by the Natural Science Foundation of Liaoning Province (No 20170541041) and the Fund for Scientific Research of The First Hospital of China Medical University (No FSFH201712).

\section{Author contributions}

All authors contributed toward data analysis, drafting and critically revising the paper and agree to be accountable for all aspects of the work. 


\section{Disclosure}

The authors report no conflicts of interest in this work.

\section{References}

1. Wong WL, Su X, Li X, et al. Global prevalence of age-related macular degeneration and disease burden projection for 2020 and 2040: a systematic review and meta-analysis. Lancet Glob Health. 2014;2(2): e106-e116.

2. Cheung LK, Eaton A. Age-related macular degeneration. Pharmacotherapy. 2013;33(8):838-855.

3. Prokofyeva E, Zrenner E. Epidemiology of major eye diseases leading to blindness in Europe: a literature review. Ophthalmic Res. 2012; 47(4):171-188.

4. Brown GC, Brown MM, Sharma S, et al. The burden of age-related macular degeneration: a value-based medicine analysis. Trans Am Ophthalmol Soc. 2005;103:173-184.

5. Folk JC, Stone EM. Ranibizumab therapy for neovascular age-related macular degeneration. N Engl J Med. 2010;363(17):1648-1655.

6. Friedman DS, O'Colmain BJ, Muñoz B, et al. Prevalence of age-related macular degeneration in the United States. Arch Ophthalmol. 2004; 122(4):564-572.

7. Schoenberger SD, Kim SJ. Nonsteroidal anti-inflammatory drugs for retinal disease. Int J Inflam. 2013;2013:281981.

8. Brown DM, Kaiser PK, Michels M, et al. Ranibizumab versus verteporfin for neovascular age-related macular degeneration. N Engl J Med. 2006;355(14):1432-1444.

9. Kodjikian L, Decullier E, Souied EH, et al. Bevacizumab and ranibizumab for neovascular age-related macular degeneration: an updated meta-analysis of randomised clinical trials. Graefes Arch Clin Exp Ophthalmol. 2014;252(10):1529-1537.

10. Rosenfeld PJ, Brown DM, Heier JS, et al. Ranibizumab for neovascular age-related macular degeneration. $N$ Engl J Med. 2006;355(14): 1419-1431.

11. Peng Y, Zhang X, Mi L, et al. Efficacy and safety of conbercept as a primary treatment for choroidal neovascularization secondary to punctate inner choroidopathy. BMC Ophthalmol. 2017;17(1):87.

12. Li X, Xu G, Wang Y, et al. Safety and efficacy of conbercept in neovascular age-related macular degeneration: results from a 12-month randomized phase 2 study: AURORA study. Ophthalmology. 2014; 121(9):1740-1747.

13. Xu D, Kaiser PK. Intravitreal aflibercept for neovascular age-related macular degeneration. Immunotherapy. 2013;5(2):121-130.

14. Zhang M, Yu D, Yang C, et al. The pharmacology study of a new recombinant human VEGF receptor-fc fusion protein on experimental choroidal neovascularization. Pharm Res. 2009;26(1):204-210.

15. Phillips B. Towards evidence based medicine for paediatricians. Confident in predicting? Meta-analysis models, step two. Arch Dis Child. 2011;96(12):1199.

16. Clarke M, Horton R. Bringing it all together: Lancet-Cochrane collaborate on systematic reviews. Lancet. 2001;357(9270):1728.

17. Cai XA, Peng H. Efficacy of two anti-VEGF drugs for age-related macular degeneration. Guoji Yanke Zazhi. 2016;16:1501-1503.

18. Cui J, Sun D, Lu H, et al. Comparison of effectiveness and safety between conbercept and ranibizumab for treatment of neovascular age-related macular degeneration. A retrospective case-controlled non-inferiority multiple center study. Eye. 2018;32(2):391-399.

19. Huang Z, Ding Q, Yan M, et al. Short-term efficacy of conbercept and ranibizumab for polypoidal choroidal vasculopathy. Retina. 2018:1.

20. Li L, Li L, Xy M. Clinical effect of conbercept and rezumumab in the treatment of wet age-related macular degeneration. Chinese Community Doctors. 2018;34(9):14-15.

21. Lv P, Xu H, Wang Q. The comparison of clinical efficacy between conbercept and ranibizumab for treatment of nAMD. $J$ Community Med. 2016;14:30-31.
22. Niu J, Jin L, Liu X, Zhang H. Comparison of efficacy between ranibizumab and conbercept for wet age-related macular degeneration. Guangxi Med J. 2016;38:641-643.

23. Yang J. Efficacy comparison of rezumumab and conpacept in the treatment of senile wet macular degeneration. J North Sichuan Med College. 2018;33(1):108-110.

24. Zhang HX, Zhao NN. Effect of conbercept and Lucentis on serum CRP, VEGF and CMT, CNV, IOP in age-related macular degeneration. Chin J Biochem Pharm. 2016;36:134-136.

25. Zhang L. The effect analysis of conbercept and ranibizumab for the treatment of neovascular age-related macular degeneration. J Changzhi Med Coll. 2017;31:209-212.

26. Zhang ZQ, Bai NY. Curative effect of different drugs in treatment of senile wet macular degeneration. Int Eye Sci. 2017;17:1123-1126.

27. Zhao YZ, Bai HN. Comparison of efficacy between ranibizumab and conbercept for neovascular age-related macular degeneration. World Latest Med Inform. 2015;64:97-98.

28. Zheng M. The comparison of therapy effectiveness between ranibizumab and conbercept for neovascular age-related macular degeneration. J Math Med. 2017;2:228-230.

29. Zhang M, Zhang J, Yan M, et al. A phase 1 study of KH902, a vascular endothelial growth factor receptor decoy, for exudative age-related macular degeneration. Ophthalmology. 2011;118(4):672-678.

30. Inoue M, Arakawa A, Yamane S, Kadonosono K. Short-term efficacy of intravitreal aflibercept in treatment-naive patients with polypoidal choroidal vasculopathy. Retina. 2014;34(11):2178-2184.

31. Hua R, Li C, Hu Y, Chen L. The discrepancy between central foveal thickness and best corrected visual acuity in cystoid macular edema secondary to central retinal vein occlusion after intravitreal lucentis ${ }^{\circledR}$ injection. Photodiagnosis Photodyn Ther. 2015;12(2):310-313.

32. Helmy YM, Atta Allah HR. Optical coherence tomography classification of diabetic cystoid macular edema. Clin Ophthalmol. 2013;7: 1731-1737.

33. Uji A, Murakami T, Nishijima K, et al. Association between hyperreflective foci in the outer retina, status of photoreceptor layer, and visual acuity in diabetic macular edema. Am J Ophthalmol. 2012;153(4): 710-717.

34. Bringmann A, Reichenbach A, Wiedemann P. Pathomechanisms of cystoid macular edema. Ophthalmic Res. 2004;36(5):241-249.

35. Pelosini L, Hull CC, Boyce JF, Mchugh D, Stanford MR, Marshall J. Optical coherence tomography may be used to predict visual acuity in patients with macular edema. Invest Ophthalmol Vis Sci. 2011;52(5): 2741-2748.

36. Beazley-Long N, Hua J, Jehle T, et al. VEGF-A165b is an endogenous neuroprotective splice isoform of vascular endothelial growth factor A in vivo and in vitro. Am J Pathol. 2013;183(3):918-929.

37. Foxton RH, Finkelstein A, Vijay S, et al. VEGF-A is necessary and sufficient for retinal neuroprotection in models of experimental glaucoma. Am J Pathol. 2013;182(4):1379-1390.

38. Mantelli F, Lambiase A, Colafrancesco V, Rocco ML, Macchi I, Aloe L. NGF and VEGF effects on retinal ganglion cell fate: new evidence from an animal model of diabetes. Eur J Ophthalmol. 2014;24(2): 247-253.

39. Chen Y, Hua R. Therapeutic Efficacy of Conbercept for Inflammatory Choroidal Neovascularization. J Ocul Pharmacol Ther. 2018; 34(3):235-236.

40. Qu J, Cheng Y, Li X, Yu L, Ke X, AURORA Study Group. Efficacy of intravitreal injection of conbercept in polypoidal choroidal vasculopathy: subgroup analysis of the aurora study. Retina. 2016;36(5):926-937.

41. Lu X, Sun X. Profile of conbercept in the treatment of neovascular age-related macular degeneration. Drug Des Devel Ther. 2015;9: 2311-2320.

42. de Oliveira Dias JR, de Andrade GC, Novais EA, Farah ME, Rodrigues EB. Fusion proteins for treatment of retinal diseases: aflibercept, ziv-aflibercept, and conbercept. Int J Retina Vitreous. 2016;2:3. 
43. Lu X, Sun X. Profile of conbercept in the treatment of neovascular age-related macular degeneration. Drug Des Devel Ther. 2015;9 2311-2320.

44. Zhang M, Zhang J, Yan M, Li H, Yang C, Yu D. Recombinant antivascular endothelial growth factor fusion protein efficiently suppresses choridal neovasularization in monkeys. Mol Vis. 2008;14:37-49.
45. Holash J, Davis S, Papadopoulos N, et al. VEGF-Trap: a VEGF blocker with potent antitumor effects. Proc Natl Acad Sci U S A. 2002;99(17): 11393-11398.

\section{Publish your work in this journal}

Drug Design, Development and Therapy is an international, peerreviewed open-access journal that spans the spectrum of drug design and development through to clinical applications. Clinical outcomes, patient safety, and programs for the development and effective, safe, and sustained use of medicines are the features of the journal, which has also been accepted for indexing on PubMed Central. The manuscript management system is completely online and includes a very quick and fair peer-review system, which is all easy to use. Visit http://www.dovepress.com/testimonials.php to read real quotes from published authors.

Submit your manuscript here: http://www.dovepress.com/drug-design-development-and-therapy-journal 